\title{
Operator on Hilbert space and its application to certain multivalent functions with fixed point associated with hypergeometric function
}

\author{
A. M. Gbolagade, D. O. Makinde \\ ${ }^{1}$ Department of Mathematics, Emmanuel Alayande College of Education, P. M. B. 1010, Oyo, Oyo State, Nigeria \\ ${ }^{2}$ Department of Mathematics, Obafemi Awolowo University, Ile-Ife, Nigeria \\ E-mail: gbolagadeam@yahoo. $\mathrm{com}^{1}$, funmideb@yahoo. $\mathrm{com}^{2}$
}

\begin{abstract}
By applying hypergeometric operator on Hilbert space, the author introduces a new class of meromorphic multivalent functions with an arbitrary fixed point omega. Properties such as coefficient inequalities, distortion bounds and extreme points were derived. Furthermore, the effect of this operator on functions in this class was also investigated.
\end{abstract}

2010 Mathematics Subject Classification. 30C45. 30C50

Keywords. Hilbert space, multivalent functions, analytic functions, univalent functions, Hadamard product, hypergeometric functions.

\section{Introduction}

Let $A$ denote the class of functions of the form

$$
f(z)=z+\sum_{k=2}^{\infty} a_{k} z^{k}
$$

which are analytic in the unit disk $U=\{z:|z|<1\}$ and normalized with $f(0)=f^{\prime}(0)-1=0$. Several reseachers have studied various aspects of this function and they came out with many interesting results as it contained in many existing literatures.

In the recent past, Kanas and Ronning [11], precisely in 1999 introduced and studied a new subclass of $A$ denoted by $A(\omega)$ with the function of the form

$$
f(z)=(z-\omega)+\sum_{k=2}^{\infty} a_{k}(z-\omega)^{k}
$$

which are analytic in the unit disk $U=\{z:|z|<1\}$ and normalized with $f(\omega)=f^{\prime}(\omega)-1=0$ where $\omega$ is an arbitrary fixed point in $U$. Authors like Acu and Owa [1], Oladipo et al [13], Oladipo $[14,15]$, Aouf et al [3] have studied various aspect of this class of functions and they obtained many interesting results. The works of previous authors mentioned above serve as our motivation to introduce and study a new class of multivalent function in this direction. We denote by $S(\omega)$ all functions in $A(\omega)$ that are univalent, where $A(\omega) \subset A$ and $S(\omega) \subset S$.

Let $\omega$ be an arbitrary fixed point in $U$ and $\Phi(\omega)$ denotes the class of functions $f(z)$ of the form

$$
f(z)=\frac{B}{(z-\omega)^{p}}+\sum_{k=p}^{\infty} a_{k}(z-\omega)^{k},(p \in N=1,2, . .)
$$


where $B$ is the residue of $f(z)$ in $(z-\omega), 0<B \leq 1$.

Also, let $T_{\omega}(p)$ denotes the subclass of $\Phi_{\omega}(p)$ consisting of functions of the form

$$
f(z)=\frac{B}{(z-\omega)^{p}}-\sum_{k=p}^{\infty} a_{k}(z-\omega)^{k},(z \in U, p \in N=1,2, . .)
$$

For the function $f(z) \in T_{\omega}(p)$, the author consider the operator $I_{p}^{n}$ as follows

$$
\begin{gathered}
I_{p}^{0} f(z)=f(z) \\
I_{p}^{1} f(z)=(z-\omega) f^{\prime}(z)+\frac{2 B p}{(z-\omega)^{p}} \\
I_{p}^{n} f(z)=(z-\omega)\left(I_{p}^{n-1} f(z)\right)^{\prime}+\frac{2 B p^{n}}{(z-\omega)^{p}}
\end{gathered}
$$

and thus

$$
I_{p}^{n} f(z)=\frac{B p^{n}}{(z-\omega)^{p}}+\sum_{k=p}^{\infty} k^{n} a_{k}(z-\omega)^{k}
$$

Now, if $f(z)$ is of the form (4), we define the basic hypergeometric function for complex parameters $a_{1}, \ldots, a_{r}$ and $b_{1}, \ldots, b_{s}$ by

$$
{ }_{r} F_{s}\left(a_{1}, \ldots, a_{r} ; b_{1}, \ldots, b_{s} ; q, z\right)=\sum_{k=o}^{\infty} \frac{\left(a_{1}, q\right)_{k} \ldots\left(a_{r}, q\right)_{k}}{(q, q)_{k}\left(b_{1}, q\right)_{k} \ldots\left(b_{s}, q\right)_{k}}(z-\omega)^{k}
$$

$\left(r=s+1 ; r, s \in N_{o}=0,1,2 . . ; z \in U\right)$, where $N$ denotes the set of positive integers and $(a, q)_{k}$ is the q-shifted factorial defined by

$$
(a, q)_{k}=\left\{\begin{array}{l}
1, k=0 \\
(1-a)(1-a q)\left(1-a q^{2}\right) \ldots\left(1-a q^{k-1}\right), k \in N
\end{array}\right.
$$

where ${ }_{r} F_{s}\left(a_{1}, \ldots, a_{r} ; b_{1}, \ldots, b_{s}, z\right)$ is the well known generalized hypergeometric function. For further background information on basic hypergeometric function, see [2], [5], [8].

Corresponding to the function ${ }_{r} F_{s}\left(a_{1}, \ldots, a_{r} ; b_{1}, \ldots, b_{s}, q, z\right)$, consider

$$
\begin{gathered}
{ }_{r} \Omega_{s}\left(a_{1}, \ldots, a_{r} ; b_{1}, \ldots, b_{s}, q, z, \omega\right)=\frac{B}{(z-\omega)^{p}}{ }_{r} F_{s}\left(a_{1}, \ldots, a_{r} ; b_{1}, \ldots, b_{s}, q, z\right) \\
=\frac{B}{(z-\omega)^{p}}-\sum_{k=p}^{\infty} \frac{\left(a_{1}, q\right)_{k} \ldots\left(a_{r}, q\right)_{k}}{(q, q)_{k} \ldots\left(b_{1}, q\right) \ldots\left(b_{s}, q\right)_{k}}(z-\omega)^{k}
\end{gathered}
$$


where $p \in N=1,2, .$. and $0<B \leq 1$

The author wish to define a linear operator $T_{s}^{r}(\omega)\left(a_{1}, \ldots, a_{r} ; b_{1}, \ldots, b_{s}, q, \omega\right) f: A(\omega) \rightarrow A(\omega)$ by

$$
\begin{gathered}
\rho(z)=T_{s}^{r}\left(a_{1}, \ldots a_{r} ; b_{1}, \ldots b_{s} ; q, \omega\right) f={ }_{r} \Omega_{s}\left(a_{1}, \ldots, a_{r} ; b_{1}, \ldots, b_{s}, q, z, \omega\right) * f(z) \\
=\frac{B p^{n}}{(z-\omega)^{p}}-\sum_{k=p}^{\infty} \Gamma(a, q, k) k^{n} a_{k}(z-\omega)^{k}
\end{gathered}
$$

where

$$
\Gamma(a, q, k)=\frac{\left(a_{1}, q\right)_{k} \ldots\left(a_{r}, q\right)_{k}}{(q, q)_{k} \ldots\left(b_{1}, q\right) \ldots\left(b_{s}, q\right)_{k}}
$$

$n=0,1,2 . ., p \in N=1,2, . .$, and $*$ denotes the Hadamard product (or convolution) of ${ }_{r} \Omega_{s}$ and $f$. Definition A. The function $\rho(z) \in T_{\omega}(p)$ is said to be a member of the class $T_{\omega}^{n}(\alpha, \beta, \gamma, p)$ if it satisfies

$$
\left|\frac{(z-\omega)^{3}(\rho(z))^{\prime \prime}+(z-\omega)^{2}(\rho(z))^{\prime}-B p^{n}}{2(z-\omega)(\rho(z))-\beta(1+\gamma) B p^{n}}\right|<\alpha
$$

where $\alpha, \beta, \gamma \in[0,1)$ and $\omega$ is an arbitrary fixed point in $U$. Let $H$ be a Hilbert space on $C$ and $\mu$ be a linear operator on $H$. Also, let $f(\mu)$ be the operator on $H$ defined by modified Riesz-Dunford integral [4]

$$
2 \prod i f(\mu)=\int_{c} f(z)((z-\omega) I-\mu)^{-1} d z
$$

where $c$ is a positively oriented simple close rectifiable contour lying in $U=\{z:|z|<1\}$ and which also contain the spectrum of $\mu$ in its interior domain and $I$ is the identity operator on $H$. see [6], [7].

Definition B. A function $\rho(z)$ given by (9) is in the class $T_{\omega}^{n}(\alpha, \beta, \gamma, p, \mu)$ if for all operator $\mu$ with $\|\mu\|<1$ and $\mu \neq 0$, it satisfies the inequality

$$
\left\|\mu^{3}\left[I_{p} f(\mu)\right]^{\prime \prime}+\mu^{2}\left[I_{p} f(\mu)\right]^{\prime}-B p^{n+2}\right\| \leq \alpha\left\|-2 \mu I_{p} f(\mu)-\beta(1+\gamma) B p^{n}\right\|
$$

where $\alpha, \beta, \gamma \in[0,1)$ Recently, Ghanim and Darus [9], Joshi [10], Xiaopei [16] and Najafzadeh et al [12] considered the operator on Hilbert space and they obtained useful and interesting results.

\section{Main result}

Here, the author obtain coefficient inequalities and distortion property for a function $\rho(z) \in$ $T_{\omega}^{n}(\alpha, \beta, \gamma, p, \mu)$

Theorem 1. A function $\rho(z)$ given by (9) is in the class $T_{\omega}^{n}(\alpha, \beta, \gamma, p, \mu)$ for all $\mu=0$ if and only if

$$
\sum_{k=p}^{\infty} \frac{\Gamma(a, q, k)\left(k^{n+2}+2 \alpha\right)}{\alpha B p^{n}[2-\beta(1+\gamma)]} a_{k} \leq 1
$$


The result is sharp for the function $\rho(z)$ given by

$$
M(z)=\frac{B}{(z-\omega)^{p}}-\frac{\alpha B p^{n}[2-\beta(1+\gamma)]}{\Gamma(a, q, k)\left(k^{n+2}+2 \alpha\right)}(z-\omega)^{k}
$$

for $k \geq 1$ and $p \in N=1,2,3, .$.

Proof. Supposing that (13) holds true, we have

$$
\begin{gathered}
\left\|\mu^{3} f^{\prime \prime}(\mu)+\mu^{2} f^{\prime}(\mu)-B p^{n+2}\right\|-\alpha\left\|2 \mu f(\mu)-\beta(1+\gamma) B p^{n}\right\| \\
=\left\|-\sum_{k=p}^{\infty} \Gamma(a, q, k) k^{2} a_{k} \mu^{n+p}\right\|-\alpha\left\|B p^{n}[2-\beta(1+\gamma)]-\sum_{k=p}^{\infty} 2 \Gamma(a, q, k) k^{n} a_{k} \mu^{n+p}\right\| \\
\leq \sum_{k=p}^{\infty} \Gamma(a, q, k)\left(k^{2}+2 \alpha\right)-\alpha B p^{n}[2-\beta(1+\gamma)] \leq 0
\end{gathered}
$$

Hence, $\rho$ is in the class $T_{\omega}^{n}(\alpha, \beta, \gamma, p, \mu)$

Conversely, supposing that

$$
\left\|\mu^{3} f^{\prime \prime}(\mu)+\mu^{2} f^{\prime}(\mu)-B p^{n+2}\right\|<\alpha\left\|2 \mu f(\mu)-\beta(1+\gamma) B p^{n}\right\|
$$

such that

$$
\left\|-\sum_{k=p}^{\infty} \Gamma(a, q, k) k^{n+2} a_{k} \mu^{n+p}\right\|<\alpha\left\|B p^{n}[2-\beta(1+\gamma)]-\sum_{k=p}^{\infty} 2 \Gamma(a, q, k) k^{n} a_{k} \mu^{n+p}\right\|
$$

On setting $\mu=\mu I(0<\mu<1)$ in (18), we obtain

$$
\frac{\sum_{k=p}^{\infty} \Gamma(a, q, k) k^{n+2} a_{k} \mu^{n+p}}{B p^{n}[2-\beta(1+\gamma)]-\sum_{k=p}^{\infty} 2 \Gamma(a, q, k) k^{n} a_{k} \mu^{n+p}}<\alpha
$$

By letting $\mu \rightarrow 1$ then (19) becomes

$$
\sum_{k=p}^{\infty} \Gamma(a, q, k) k^{n+2} a_{k}<\alpha B p^{n}[2-\beta(1+\gamma)]-\sum_{k=p}^{\infty} 2 \alpha \Gamma(a, q, k) k^{n} a_{k}
$$

or simply written as

$$
\sum_{k=p}^{\infty} \Gamma(a, q, k)\left(k^{n+2}+2 \alpha\right) a_{k} \leq B p^{n}[2-\beta(1+\gamma)]
$$

and this complete the proof.

Corollary. If $\rho(z)$ given by (9) be in the class $T_{\omega}^{n}(\alpha, \beta, \gamma, p, \mu)$, then

$$
a_{k} \leq \frac{B p^{n}[2-\beta(1+\gamma)]}{\Gamma(a, q, k)\left(k^{n+2}+2 \alpha\right)}, k, p \in N
$$


Theorem 2. If $\rho(z)$ of the form (9) is in the class $T_{\omega}^{n}(\alpha, \beta, \gamma, p, \mu),\|\mu\| \leq 1$ and $\mu \neq 0$, then

$$
\begin{gathered}
\left\|\frac{B}{\mu^{p}}\right\|-\frac{\alpha B p^{n}[2-\beta(1+\gamma)]}{\Gamma(a, q, k)\left(k^{n+2}+2 \alpha\right)}\|\mu\|^{k} \leq\|f(\mu)\| \\
\quad \leq\left\|\frac{B}{\mu^{p}}\right\|+\frac{\alpha B p^{n}[2-\beta(1+\gamma)]}{\Gamma(a, q, k)\left(k^{n+2}+2 \alpha\right)}\|\mu\|^{k}
\end{gathered}
$$

The result is sharp for the function $M(z)$ given by (14)

Proof. By theorem 1, we have

$$
\sum_{k=p}^{\infty} a_{k} \leq \frac{\alpha B p^{n}[2-\beta(1+\gamma)]}{\Gamma(a, q, k)\left(k^{n+2}+2 \alpha\right)}
$$

and

$$
\|f(\mu)\| \geq\left\|\frac{B}{\mu^{P}}\right\|-\|\mu\|^{k} \sum_{k=p}^{\infty} a_{k}
$$

hence, we obtain

$$
\geq\left\|\frac{B}{\mu^{p}}\right\|-\frac{\alpha B p^{n}[2-\beta(1+\gamma)]}{\Gamma(a, q, k)\left(k^{n+2}+2 \alpha\right)}\left\|\mu^{k}\right\|
$$

and

$$
\begin{gathered}
\|f(\mu)\| \leq\left\|\frac{B}{\mu^{P}}\right\|+\|\mu\|^{k} \sum_{k=p}^{\infty} a_{k} \\
\leq\left\|\frac{B}{\mu^{p}}\right\|+\frac{\alpha B p^{n}[2-\beta(1+\gamma)]}{\Gamma(a, q, k)\left(k^{n+2}+2 \alpha\right)}\left\|\mu^{k}\right\|
\end{gathered}
$$

and the proof is complete

Next, the author discuss about extreme point of $T_{\omega}^{n}(\alpha, \beta, \gamma, p, \mu)$ and effect of operator on functions in this class

Theorem 3. Let $\rho_{o}=\frac{B}{(z-\omega)^{p}}$ and $\rho_{n}(z)=\frac{B}{(z-\omega)^{p}}-\frac{\alpha B p^{n}[2-\beta(1+\gamma)]}{\Gamma(a, q, k)\left(k^{n+2}+2 \alpha\right)}(z-\omega)^{k}$, then $\rho(z) \in$ $T_{\omega}^{n}(\alpha, \beta, \gamma, p, \mu)$ if and only if it can be expressed as $\rho(z)=\sum_{k=0}^{\infty} \lambda_{k} \rho_{k}(z)$ where $\lambda \geq 0$ and $\sum_{k=0}^{\infty} \lambda_{k}=1$

Proof. Let $\rho(z)=\sum_{k=0}^{\infty} \lambda_{k} \rho_{k}(z)$

$$
=\frac{B}{(z-\omega)^{p}}-\sum_{k=p}^{\infty} \lambda_{k} \frac{\alpha B p^{n}[2-\beta(1+\gamma)]}{\Gamma(a, q, k)\left(k^{n+2}+2 \alpha\right)}(z-\omega)^{k}
$$

since

$$
\sum_{k=p}^{\infty} \frac{\Gamma(a, q, k)\left(k^{n+2}+2 \alpha\right)}{\alpha B p^{n}[2-\beta(1+\gamma)]} X \frac{\alpha B p^{n}[2-\beta(1+\gamma)]}{\Gamma(a, q, k)\left(k^{n+2}+2 \alpha\right)} \lambda_{k}=\sum_{k=p}^{\infty} \lambda_{k}=1-\lambda_{o} \leq 1
$$


So, by theorem 1 , we find that $\rho(z) \in T_{\omega}^{n}(\alpha, \beta, \gamma, p, \mu)$.

Conversely, suppose that $\rho(z) \in T_{\omega}^{n}(\alpha, \beta, \gamma, p, \mu)$, then by (22) we have

$$
a_{k} \leq \frac{\alpha B p^{n}[2-\beta(1+\gamma)]}{\Gamma(a, q, k)\left(k^{n+2}+2 \alpha\right)}
$$

Setting

$$
\lambda_{k}=\frac{\Gamma(a, q, k)\left(k^{n+2}+2 \alpha\right)}{\alpha B p^{n}[2-\beta(1+\gamma)]} a_{k}
$$

and $\lambda_{o}=1-\sum_{k=p}^{\infty} \lambda_{k}$ and the proof completes

Theorem 4. If $\rho(z) \in T_{\omega}^{n}(\alpha, \beta, \gamma, p, \mu)$, then the function $F_{c, p}(z)$ defined by $F_{c, p}=c \int_{0}^{1}\left[s^{c} \rho(z s+\right.$ $\omega(1-s))] d s, c \geq 1$,is also in this class.

Proof. Since $\rho(z) \in T_{\omega}^{n}(\alpha, \beta, \gamma, p, \mu)$ is of the form (9), so

$$
\begin{gathered}
F_{c, p}=c \int_{d}^{1} s^{c}\left[\frac{B}{s(z-\omega)^{p}}-\sum_{k=p}^{\infty} \Gamma(a, q, k) k^{n} a_{k}(s(z-\omega))^{k}\right] d s, d<1 \\
=\frac{B}{(z-\omega)^{p}}-\sum_{k=p}^{\infty} \Gamma(a, q, k) k^{n} \frac{c}{c+k+1} a_{k}(z-\omega)^{k}
\end{gathered}
$$

$\frac{c}{c+k+1}<1$, hence by theorem 1 , we conclude that

$$
F_{c, p}(z) \in T_{\omega}^{n}(\alpha, \beta, \gamma, p, \mu)
$$

\section{References}

[1] Acu Mugur and Shigeyoshi Owa, On some subclasses of univalent functions, Journal of Inequalities in Pure and Applied Mathematics, 6, No. 3, Article 70 (2005), 1-14.

[2] H. Aldweby and M. Darus, A subclass of hamonic univalent functions associated with q-analogue of Dziok-Srivastava operator, ISRN Math. Anal.2013, Art. ID 382312, 6pp.

[3] M. K. Aouf, A. Shamandy, A. O. Mostafa and S. M. Madian, A subclass of $m-\omega$ starlike functions, Acta Universitatis Apulensis, No. 21, (2010), 135-142.

[4] R. Dunford and J. T. Schwartz, Linear operators, part 1, General Theory, New York - London,Interscience, 1985.

[5] H. Exton, q-hypergeometric functions and applications, Ellis Horwood Series: Mathematics and its application, Ellis Horwood, Chichester, UK, 1983.

[6] F. Fan, Analytic functions of a proper contractions, Math. Z, 160, (1978), 275-290. 
[7] B. A. Frasin and M. Darus, On certain meromorphic functions with positive coefficients, South East Asian Bull. of Math., 28, (2004), 615-623.

[8] G. Gasper and M. Rahman, Basic hypergeometric series, Vol. 35 of Encyclopedia of Mathematics and its applications, Cambridge Unlversity Press, Cambridge, UK, 1990.

[9] F. Ghanim and M. Darus, On new subclass of analytic p-valent functions with negative coefficient for operator on Hilbert space, Int. Math. Forum, 3, No. 2 (2008), 69-77.

[10] S. B. Joshi, On a class of analytic functions with negative coefficient for operator on Hilbert space, J. App. Theory and Application, (1998), 107-112.

[11] S. Kanas and F. Ronning, Uniformly starlike and convex functions and other related classes of univalent functions, Ann. Univ. Mariae Curie-skldowska Section A, 53, (1999), 95-105.

[12] SH. Najafzadeh and A. Ebadian, Operator on Hilbert space and its application to certain univalent function with a fixed point, Acta Universitatis Apulensis, No. 27 (2011), 51-56.

[13] A. T. Oladipo, A. O. Fadipe-Joseph and B. O. Moses, On a new class of uniformly analytic functions associated with q-analogue of Dziok-Srivastava operator, Far East Journal of Mathematical Sciences, 89, No. 2 (2014), 153-167.

[14] A. T. Oladipo, On certain classes of analytic and univalent functions involving convolution operators, Acta Universitatis Apulensis, Math. Inform., No. 20 (2009), 163-174.

[15] A. T. Oladipo, On subclass of analytic and univalent functions, Advances in Applied Mathematical Analysis, 4, No. 1 (2009), 87-93.

[16] Y. Xiaopei, A subclass of analytic p-valent functions for operator on Hilbert space, Math. Japan, 40, (1984), 303-308. 